\begin{tabular}{|l|l|l|}
\hline TABLE 1 & & \\
\hline & OR & $p$ \\
\hline SELENA-SLEDAI & $1.10(1.06-1.14)$ & $<0.001$ \\
\hline SLICC/ACR DI & $1.27(1.16-1.38)$ & $<0.001$ \\
\hline Elevated creatinine & $2.08(1.66-2.61)$ & $<0.001$ \\
\hline Active nephritis & $3.52(1.94-6.37)$ & $=0.001$ \\
\hline Hepatitis C & $4.82(1.89-12.27)$ & $=0.002$ \\
\hline Diabetes & $3.87(2.06-7.26)$ & $=0.0001$ \\
\hline Cancer & $3.60(2.01-6.42)$ & $=0.000$ \\
\hline Corticosteroids (Prednisone > 10mg/day) & $1.81(1.07-3.09)$ & $=0.023$ \\
\hline Immunosuppressors & $11.44(7.31-17.92)$ & $=0.000$ \\
\hline Antimalarials & $0.39(0.25-0.61)$ & $=0.000$ \\
\hline Renal Transplant & $5.64(2.63-12.1)$ & $=0.000$ \\
\hline Dialysis & $0.39(0.25-0.61)$ & $=0.000$ \\
\hline
\end{tabular}

recurrence and mortality were high. Immunosuppressors use, comorbidity and damage were all associated to bacteraemia.

Disclosure of Interest: None declared

DOI: 10.1136/annrheumdis-2017-eular.4138

\section{OP0043 THE NUMBER OF CIRCULATING REGULATORY T CELLS IS REDUCED AND LOW-DOSE IL-2 SELECTIVELY STIMULATES ITS PROLIFERATION IN PATIENTS WITH SYSTEMIC LUPUS ERYTHEMATOSUS}

X. Liu ${ }^{1}$, X. Jing ${ }^{1}$, X. Wu ${ }^{1}$, J. Wang ${ }^{1}$, Z. Liang ${ }^{1}$, M. Hao ${ }^{1}$, J. Chen ${ }^{1}$, C. Gao ${ }^{2}$, X. Li ${ }^{1} .{ }^{1}$ Rheumatology, The Second Hospital of Shanxi Medical University, Taiyuan, China; ${ }^{2}$ Pathology, Brigham and Women's Hospital, Harvard Medical School, Boston, United States

Background: The imbalance of $\mathrm{T}$ help 17 cells (Th17)/regulatory $\mathrm{T}$ cells (Tregs) is considered to be a pivotal cause of autoimmune diseases ${ }^{1}$, including systemic lupus erythematosus (SLE). However, previous reports ${ }^{1,2}$ describing the respective changes of Tregs and Th17 cells in SLE patients were controversial because a few samples or diverse markers were used to identify Tregs with little consensus.

Objectives: To clarify the status of Tregs and Th17 in SLE, we investigated the frequencies of Tregs and Th17 cells on a large scale and whether those defects can be corrected by the supplementation of low-dose human recombinant interleukin-2 (IL-2).

Methods: Two hundred and thirty-five SLE patients (219 women and 16 men), with mean age of $37.80 \pm 14.00$ years, were enrolled. The disease activity using European League Against Rheumatism (EULAR) criteria was judged for SLE patients with erythrocyte sedimentation rate (ESR) and SLEDAI scores. The frequencies of CD3+CD4+FOXP3+Treg cells and Th17 cells in peripheral blood from these patients were measured by flow cytometry. And low-dose IL-2 was used among 127 patients at a dosage of fifty WIU every day for five days. Immunological and clinical assessments were performed again at the end of IL-2 treatment. Ninety healthy volunteers, matched for patients' age and gender, were also included for the estimation of CD4+ T cell subsets.

Results: As compared to healthy controls (median of Treg cells: 33.09 cells/ul), the frequencies of circulating CD4+CD25+FOXP3+Treg cells were significantly decreased in SLE patients (median: 15.49 cells/ul, $P<0.001$ ). The median ratios of Th17/Tregs cells in patients were greatly higher than those of healthy volunteers $[0.42(0.19,0.88)$ vs. $0.21(0.15,0.34), P<0.001]$. There was not significantly different in circulating Th17 cell between two groups. Moreover, CD4+CD25+FOXP3+Treg cells were negatively correlated with ESR and SLEDAI score $(\mathrm{r}=-0.198, P=0.01 ; \mathrm{r}=-0.25, P=0.002)$. While no obvious correlation was seen between Th17 cells and SLEDAI score. After IL-2 therapy in SLE, there was a fourfold increase in circulating CD4+CD25+FOXP3+Treg cells $[43.73(24.08,74.22)$ vs. $11.95(7.51,20.34), P<0.001]$, whereas Th17 cells were increased slightly. The ratio of Th17/Tregs was decreased significantly in patients with IL-2 treatment $[0.19(0.09,0.41)$ vs. $0.52(0.23,0.95), P<0.001]$, tended to balance and had no difference with healthy individual $(P=0.275)$.

Conclusions: With the increase of disease activity,CD4+CD25+FOXP3+Treg cells were gradually reducing, while Th17 cells did not show a significant change, indicating that the reduction of Tregs but not the elevation of Th17 cells may be the major reason for imbalance of Th17/Tregs. It is speculated that SLE is an autoimmune disease triggered by the defect of immunotolerance. More importantly, low-dose IL-2 selectively modulated the abundance of Tregs, which effectively induced autoimmune tolerance and further improved clinical symptoms. References:

[1] Ji Y, Xue Y, Hejian Z, et al.Recovery of the immune balance betweenTh17 and regulatory $T$ cells as a treatment for systemic lupus erythematosus[J]. Rheumatology 2011;50:1366-1372.

[2] Steinmetz OM, Turner JE, Paust HJ et al. CXCR3 mediates renal Th1 and Th17 immune response in murine lupus nephritis[J]. J Immunol 2009;183:4693-704.

Disclosure of Interest: None declared

DOI: 10.1136/annrheumdis-2017-eular.4221

\section{OP0044 ANTIBODIES ANTI-SACCHAROMYCES CEREVISIAE IN PRIMARY SJÖGREN'S SYNDROME: PREVALENCE, CLINICAL ASSOCIATIONS AND POSSIBLE CROSS-REACTIVITY WITH DISEASE SPECIFIC AUTOANTIGENS}

A. Alunno ${ }^{1}$, O. Bistoni ${ }^{1}$, F. Carubbi ${ }^{2,3}$, G. Cafaro ${ }^{1}$, E. Valentini ${ }^{4}$, R. Giacomelli ${ }^{2}$ R. Gerli ${ }^{1}{ }^{1}$ Department of Medicine, Rheumatology Unit, University of Perugia, Perugia; ${ }^{2}$ Department of Biotechnological and Applied Clinical Sciences, Rheumatology Unit, University of L'Aquila; ${ }^{3}$ Department of Medicine, ASL 1 Avezzano-L'Aquila-Sulmona, L'Aquila; ${ }^{4}$ Department of Medicine, Rheumatology Unit, University di Perugia, Perugia, Italy

Background: Saccharomyces cerevisiae is a common yeast used in the food industry. Antibodies against the phosphopeptidomannan part of the cell wall of S. cerevisiae (ASCA) are a well established biomarker of Crohn's disease and have been assessed in several organ-specific and systemic autoimmune diseases (ADs) (1-2) Although the pathogenic significance of ASCA is not yet fully understood, the molecular mimicry of self-antigens in several associated ADs has been suggested as putative mechanism.

Objectives: Since to date ASCA have not been tested in primary Sjogren's syndrome (pSS), the purpose of this study was to assess these antibodies in a large cohort of pSS patients and investigate their significance as potentially helpful biomarker in a clinical setting.

Methods: One hundred and four patients with pSS according to the 2002 American European Consensus criteria and 30 healthy donors (HD) were enrolled. ASCA IgG+lgA were assessed in serum samples with ASCA screen dot (Alphadia sa/nv). T cell phenotyping was performed in paired peripheral blood samples by flow cytometry. To compare the aminoacid sequence of mannan of Saccharomyces cerevisiae and well characterized auto-antigens peculiar of pSS (52kD and 60kD Ro/SSA, La/SSB) we browsed the protein database of the National Center for Biotechnology Information (NCBI) and run the Basic Local Alignment Search Tool (BLAST).

Results: ASCA were detected in 5 out of 104 pSS patients, therefore the prevalence in our cohort is $4.8 \%$. None of the ASCA ${ }^{+}$pSS patients displayed IBD or other autoimmune conditions that could account for ASCA positivity. ASCA ${ }^{+}$ pSS patients displayed more frequently a reduction of C3 and C4 complement fractions as well as pulmonary, articular and cutaneous involvement (all $p<0.05$ ). Binary logistic regression revealed that $\mathrm{ASCA}^{+} \mathrm{pSS}$ patients display an odds ratio of $14(\mathrm{p}=0.006)$ to have cutaneous manifestations of pSS. All ASCA+ patients but only $39 \%$ of ASCA- patients displayed anti-Ro52, anti-Ro60 and anti-La autoantibodies together $(p=0.01)$. No differences concerning $T$ regulatory and Th17 cell proportion could be observed in ASCA $^{+}$compared to ASCA- pSS patients. S. cerevisiae mannan displays a consistent similarity with $52 \mathrm{kD}$ and $60 \mathrm{kD} \mathrm{Ro} / \mathrm{SSA}, \mathrm{La} / \mathrm{SSB}$ autoantigens. The highest similarity was observed when aligning the mannan with $60 \mathrm{kD}$ Ro/SSA (identities $7 / 11$, 64\%; positives $8 / 11$, $72 \%$, E value 2.2.).

Conclusions: Our study assessed for the first time ASCA IgG+lgA with a highly specific immunoblot assay in a large cohort of pSS patients, showing that ASCA positivity identifies a peculiar clinical and serological pSS phenotype. In particular, $\mathrm{ASCA}^{+}$pSS patients display anti-Ro52, anti-Ro60 and anti-La antibodies, low complement and cutaneous involvement. The high similarity between $\mathrm{S}$. cerevisiae mannan and Ro60/SSA autoantigen may suggest that: i. ASCA may bind pSS autoantigens, such as anti-Ro, as already postulated for other autoantigens (2); ii. ASCA may bind more likely Ro60 autoantigen rather than the Ro52 or La autoantigens in PSS. A possible pathogenic/prognostic significance of ASCA in pSS may therefore be speculated.

References:

[1] Inflamm Bowel Dis 2012;18:1340-1355.

[2] Clin Rev Allergy Immunol 2013; 45: 152-61.

Disclosure of Interest: None declared

DOI: 10.1136/annrheumdis-2017-eular.3163

\section{OP0045 PREDICTORS OF PERSISTENT DISEASE ACTIVITY AND PERSISTENT LONG QUIESCENCE IN SYSTEMIC LUPUS ERYTHEMATOSUS - RESULTS FROM THE HOPKINS LUPUS COHORT}

I. Giannakou ${ }^{1}$, K. Chatzidionysiou ${ }^{1}$, L. Magder ${ }^{2}$, R. van Vollenhoven ${ }^{1}$, M. Petri ${ }^{3}{ }^{1}$ ClinTRID - Unit for Clinical Therapy Research, Inflammatory Diseases, Department of Medicine, Karolinska Institutet, Stockholm, Sweden; ${ }^{2}$ University of Maryland School of Medicine; ${ }^{3}$ Division of Rheumatology, Johns Hopkins University School of Medicine, Baltimore, United States

Background: Systemic lupus erythematosus (SLE) is characterized by a diversity of disease activity.

Objectives: The aim of this study was to identify prognostic factors of persistent disease activity and persistent long quiescence using baseline demographics and clinical characteristics.

Methods: Patients enrolled in the Hopkins Lupus Cohort from 1987 to 2014, who had at least 3 visits per year during 3 years following cohort inclusion and available information on disease activity were included. Three major patterns of SLE disease activity over time (1 year intervals) based on the modified SLE Disease Activity Index have been previously described: long quiescent (LQ), chronic active (CA) and relapsing-remitting (RR) (1). Based on maintenance of the aforementioned 